\title{
Comparison of recoveries of Mycobacterium tuberculosis using the automated BACTEC MGIT 960 System and Lowenstein - Jensen medium
}

\author{
Mo. A.AL-Mazini T. Bukeet A. Abdul Kareem \\ Coll. of Sci./ Unive Basrah
}

\begin{abstract}
We examined whether the BACTEC/Mycobacxteria Growth Indicator Tube (MGIT) System alone could supplant the use of a supplemental Lowenstein-Jensen (LJ) slant for routine recovery of $M$. tuberculosis from clinical specimens . A total of 392 specimens of sputum were included in the study, collected from 196 patients. Specimens were processed with standard N-acetyl- L- Cysteine (NALC-NaOH) method, then inoculated onto BACTEC MGIT 960 and onto LJ media.The recovery rates of M.tuberculosis were $100 \%(256 / 256)$ with BACTEC MGIT 960 and 72.6\%(186/256) with LJ . The rates of contamination for each of the system were $4.8 \%$ with BACTEC MGIT 960 and 5.3\%with LJ.The TTD for $M$. tuberculosis were 11.3 days with BACTEC System and 30.8 days with LJ.The difference in TTD between smear positive and smear negative specimens for M.tuberculosis with BACTEC MGIT 960 was not statistically significant.This study shows that the BACTEC System demonstrates better sensitivity for the recovery of $M$. tuberculosis from clinical specimens.
\end{abstract}

\section{Introduction}

The Mycobacterium tuberculosis includes the four species $M$. tuberculosis, M. bovis, M. africanum, and M. microti . These species are genetically very similar, with 85 to $100 \%$ DNA-DNA relatedness (Pereira et al., 2005), but they differ in their epidemiology, M. tuberculosis causes tuberculosis in humans and animals which have contact with humans . M. africanum causes tuberculosis in humans in Africa (Ginsburg et al., 2003). M. bovis is found in a wide range of wild and domestic animals, while M. microti causes tuberculosis in voles (Murugasu and Dick, 2000).Tuberculosis is one of the important diseases worldwide with about 7.3 million new cases and 3 million deaths per year (World Health Organization,2006). The problems of tuberculosis are related by the spread of HIV infection and the increases in prevalence of drug resistance as well as multidrug - resistance strains (MDR-TB) (Cohn et al., 1997).Despite the discovery of the tubercle bacillus more than a hundred years ago, and all the advances in our knowledge of the disease made since then, tuberculosis still remains one of the major health problems, particularly in developing countries (Dye et al.,
2003). The incidence of tuberculosis is high in Basrah city and remains a serious public health issue (Rooden, 2001).Moreover, the appearance of multidrug- resistance strains of Mycobacterium tuberculosis (MDR-TB) has increased the need for rapid diagnostic methods using modern techniques (BACTEC MGIT 960 System) is an automated system for the growth and detection of mycobacteria with a capacity to incubate and continuously monitor 960 mycobacteria growth indicator tube (MGIT) every 60 minutes for increase in fluorescence (Rohner et al., 2000). Growth detection is based on the acid fast bacteria (AFB) metabolic O2 utilization and subsequent intensification of an $\mathrm{O} 2$ quenched fluorescent dye contained in a tube of modified MGIT . A series of algorithms are used to determine presumptive positivity and alert the operator to the presence and location of positi-ve tubes (Jayakumar et al., 2001). Rapid detection of drug-resistance strains of $M$. tuberculosis is essential for efficient management of tuberculosis (TB) patients. For nearly two decades the BACTEC 960 TB System has provided the most rapid method for antimicrobial susceptibility 
testing (AST) (Augustinowic - Kopec et al., 2002).Few reports have been published to evaluate the efficiency of this new system (Hanna et al., 2002). The aims of this study were to evaluate the per- formance of BACTEC MGIT 960 and to compare the recovery rate and the mean time to detection (TTD) between BACTEC MGIT 960 and LJ medium in the central TB laboratory of Basrah.

\section{Materials and Methods}

\section{1- Specimens collection :}

From a total of 220 patients submitted to the Institute of the Tuberculosis and Chest Disease-Basrah city and selected at random for a period from September 2006 to April 2007,sputum specimens was taken from all patients. All specimens were digested and decontaminated according to Standard Centers for Disease Control methods (Kent and Kubica, 1985). Specimens were processed by the sodium hydroxide and N-acetyl-L-cysteine (NaOH/NALC) method, with final concentrations of $1 \%$ for $\mathrm{NaOH}$ and 0.25 $\%$ for NALC.

\section{2- AFB smears :}

Using the sediment, smears were prepared from all specimens and examined for acid - fast bacteria (AFB). All sputum smears were stained by Ziehl Neelsen (Baron et al., 1995).

\section{3-Culture medium inoculation, incubation, and test duration :}

The remaining sediment was suspended in 1 to $2 \mathrm{ml}$ of sterile phosphate - buffered saline (PH 6.8) and vortexed for 10 minutes.This suspension was then used for cultivation on medium by inoculation $0.1 \mathrm{ml}$ of processed specimen onto the surface of an Lowenstein-Jensen (LJ)medium and incubated at $37 \mathrm{C}$ for 56 days (Collee et al.,1996). While the culture system (BACTEC MGIT 960)was done: The BACTEC MGIT 960 culture tube contains $7 \mathrm{ml}$ of Middlebrook $7 \mathrm{H} 9$ broth base ,to which was added an enrichment supplement containing oleic acid, albumin, dextrose, and catalase (BBL MGIT OADC) and an antibiotic mixture of polymyxin $\mathrm{B}$, amphotericin $\mathrm{B}$, nalidixic acid, trimethoprim, and azlocillin (BBL MGIT PANTA). After inoculation of each tube with $0.5 \mathrm{ml}$ of the processed specimen , the tubes were inocubated at $37 \mathrm{C}$ in the BACTEC MGIT 960 instrument for a period of 42 days and were monitored automatically every $60 \mathrm{~min}$ for increase of fluorescence.A series of algorithms included in the instrument is used to determine presumptive positivity and to alert the operator to the presence and location of the positive tubes by means of indicator lights positioned on the front of the instrument, tube status displayed on the video display screen. Any sample which was identified as positive was removed from the instrument, and a smear was prepared and examined for AFB. Time to detection of mycobacteria was based on the date of the earliest instrument positivity, which correlated with sputum smear positivity (Hanna et al., 2002; ChunEng et al.,2004).Descriptive statistics expressed as mean and percentage were used to analyze the results for most of this study $\mathrm{t}-$ test with Excel software was used to compare between method used the Lowenstein-Jensen medium and the BACTEC MGIT 960 System .

\section{Results}

Of the 220 patients tested, 196 patients were infected with pulmonary tuberculosis. A total of 392 clinical specimens were tested, of which $256(65.3 \%)$ were smear - positive and 136 (34.6\%) were smearnegative (Table-1). Of the 256 isolates recovered, $100 \%(256 / 256)$ were recovered in BACTEC MGIT 960 and $72.6 \%$ $(186 / 256)$ on Lowenstein - Jensen medium (Table -2). The rates of smear - negative but culture - positive specimens were 24.2\%(33/136) with BACTEC MGIT 960 and $14.7 \%(20 / 136)$ with LJ medium . The contamination rates with BACTEC MGIT 960 and LJ were 19/392 (4.8\%) and 21 / 392 (5.3\%), respectively (Table-2).The numbers and rates of recovery for Mycobacterium tuberculosis and contamination are shown in Table (2). 
Table (1): The percentage between smear positive and smear negative isolates of sputum samples .

\begin{tabular}{|c|c|c|}
\hline Number of specimens & No.(\%) of positive smear & No.(\%) of negative smear \\
\hline 392 & $256(65.3 \%)$ & $136(34.6 \%)$ \\
\hline
\end{tabular}

Table (2): Numbers and rates of contamination for M.tuberculosis

\begin{tabular}{|c|c|c|}
\hline medium & Positive culture n (\%) & Contamination $\mathrm{n}(\%)$ \\
\hline BACTEC MGIT 960 & $256(100 \%)$ & $19(4.8 \%)$ \\
\hline Lowenstein - Jensen & $186(72.6 \%)$ & $21(5.3 \%)$ \\
\hline Total & 256 & 392 \\
\hline
\end{tabular}

The mean (range) time to detection (TTD) of M.tuberculosis was 11.3 (1-42) days in BACTEC MGIT 960 and 30.8 (18-
56) days on LJ medium, are shown in Table (3).

Table ( 3 ) : Explain the TTD for M. tuberculosis in both culture systems .

\begin{tabular}{|l|c|c|c|}
\hline \multirow{2}{*}{$\begin{array}{c}* \\
\text { isolates }\end{array}$} & Number of isolates & TTD & T T D \\
\cline { 3 - 4 } & & MGIT & L J \\
\hline M.tuberculosis & 289 & 11.3 & 30.8 \\
\hline
\end{tabular}

TTD: Time of detection; MGIT: BACTEC MGIT 960 ;LJ : Lowenstein - Jensen medium.

For smear positive specimens, the mean time to detection was $10.3(1-42$ days) for $M$. tuberculosis in the BACTEC MGIT 960 . On L J medium, the mean time for smear positive specimens was 29.5 (18-56 days) for M.tuberculosis. For smear negative specimens, the mean time to detection was 12.9 (4-35days) for $M$. tuberculosis is BACTEC MGIT 960 . On LJ medium, the mean time for smear negative specimens was 39.8 (17- 56 days) for M.tuberculosis The difference in TTD between smear positive and smear negative specimens for this bacteria with BACTEC MGIT 960 was statistically not significant, this results were summarized in Table (4).

Table (4):Explain TTD of M. tuberculosis between smear positive and smear negative isolates.

\begin{tabular}{|c|c|c|}
\hline \multirow{2}{*}{ medium } & T T D of $M$. & tuberculosis \\
\cline { 2 - 3 } & AFB for smear positive & AFB for smear negative \\
\hline MGIT & 10.3 ( 1-42 days $)$ & 12.9 ( 4-35 days $)$ \\
\hline L J & $29.5(18-56$ days $)$ & 39.8 ( 17-56 days $)$ \\
\hline
\end{tabular}

TTD: Time to detection (in days). AFB: acid-fast bacilli.MGIT: BACTEC MGIT 960 . LJ : Lowenstein - Jensen medium

In the other side, the relationship between infection the patients with pulmonary tuberculosis and their ages were determined. In the table (5), we showed the class of ages (25-34 and 35- 44) years were more infected with T.B compared with another classes $(\mathrm{P}<0.05)$. 
Table (5): Explain the relationship of ages patients with infection in T.B.

\begin{tabular}{|c|c|c|c|c|}
\hline NO . & Class( years $)$ & Males & Females & Total ( \%) \\
\hline 1 & $0-14$ & 2 & 0 & $2(1 \%)$ \\
\hline 2 & $15-24$ & 18 & 11 & $29(14.7 \%)$ \\
\hline 3 & $25-34$ & 50 & 15 & $65(33.1 \%)$ \\
\hline 4 & $35-44$ & 39 & 11 & $50(25.5 \%)$ \\
\hline 5 & $45-54$ & 24 & 10 & $34(17.3 \%)$ \\
\hline 6 & $55-64$ & 7 & 3 & $10(5.1 \%)$ \\
\hline 7 & Age $>65$ & 4 & 2 & $6(3 \%)$ \\
\hline Total & & 144 & 52 & 196 \\
\hline
\end{tabular}

\section{Discussion}

Despite the discovery of the tubercle bacillus more than a hundred years ago , and all the advances in our knowledge of the disease made since then, tuberculosis still remains one of the major health problems facing mankind, particulary in developing countries. Presently about one third of the worlds population is infected with Mycobacterium tuberculosis and more people die of tuberculosis than from any other infectious disease (Dye et al., 2003). Therefore, the earlier identification for $M$. tuberculosis and activity treatment were basic factors to control tuberculosis (Cruciani et al., 2004). The present study showed that the instrument of Automated BACTEC MGIT 960 System for diagnosis of $M$. tuberculosis in respiratory specimens more sensitive when compare with the conventional Lowenstein -Jensen media through the following points : of the total 256 isolates detected as smear positive in this study, only $72.6 \%$ (186/ 256) specimens were detected by LJ media , but up to $100 \%(256 / 256)$ were detected by BACTEC MGIT 960. In other world, BACTEC MGIT 960 can increase the recovery of $M$. tuberculosis. Another advantage of BACTEC System is the rapidity of growth detection.The TTD of this bacterium with BACTEC System (11.3 days) was much faster than that on LJ (30.8 days). The TTD study was somewhat biased in favor of BACTEC System, in that it was read every 60 minute while LJ were read mostly once a week.In addition, the TTD of the BACTEC MGIT 960 for smear positive specimens $(10.3$ days) was shorter than that of smear negative specimens(12.9 days).This difference was not statistically significant $(\mathrm{P}<0.05)$. This TTD for $M$. tuberculosis was shorter than reported by (Tazawa et al.1998) 13.5 days , Kobayashi et al.,1999) 14.1 days and(Hanna et al,2002) 14.4 days.The rates of smear negative but culture positive mycobacterial specimens in BACTEC MGIT 960 (21.2\%) were higher than those on LJ medium (10.9\%), again confirming the greater sensitivity of BACTEC MGIT 960 over LJ in detecting $M$. tuberculosis. The rate of contamination with BACTEC MGIT 960 was $4.8 \%$. One study indicated an initial contamination rate in MGIT of $29 \%$, which was reduced to $12 \%$ with changes in specimen processing(Cornfield et al., 1996).It was concluded that these wide variations might reflect the dissimilar conditions of specimen quality, transport time and conditions among the sites.As mentioned earlier, specimens in the present study were processed within 24 hours; this might be one of the reasons why the contamination rate with BACTEC MGIT 960 reached acceptable levels.In conclusion, the BACTEC System has been proven a valuable alternative to the conventional Lowenstein-Jensen medium for the detection of $M$. tuberculosis. In addition, this system has the additional advantages of being a fully automated, high capacity, non-radiometric system, which still has good performance characteristics.In the other side, we showed significant increased in the cases of 
pulmonary tuberculosis in the ages of 2544 years $(\mathrm{P}<0.05)$ (Table-5), this result is similar to epidemiology of pulmonary tuberculosis in the world (WHO,2006).

\section{References}

Augustinowic - Kopec, E., Jaworsky, A., and Zwolska, Z. (2002). Evaluation of the BACTEC MGIT 960 fluorescent method in diagnosis of tuberculosis. Pneumonol . Alergol. Pol.70, 450 -457 .

Baron , E.J., Peterson , L.R., and Finegold , S.M. (1995). Bailey and Scotts. Diagnostic Microbiology $.8^{\text {th }}$ ed. C.V.Mosby Co. Toronto, London.

Chun - Eng , L ., Chang - Hua , C., Ju Hua, H., Tzuu - Guang ,y., and Ren - Wen, T. ( 2004). Drug resistance of Mycobacterium tuberculosis complex in central Taiwan.J. Microbiol. Immunol. Infect. 37, $295-300$.

Cohn , D., Bustreo, F., and Raviglione, M. (1997). Drug-resistance tuberculosis; Review of the worldwide situation and the WHO/ IUATLD Global surveillance project. Clin Infect Dis . $24,121-130$.

Cornfield, D., Beavis , K.,Greene, J., Bojak, M.,and Bondi,J.(1996). Mycobacterial growth and bacterial contamination in the Mycobacterial Growth Indicator Tube and BACTEC 460 culture systems. J. Clin . Microbiol. 35, $2068-2071$.

Cruciani , M ., Scarparo, C., Malena , M., Bosco,O., Serpelloni, G., and Mengoli , C. ( 2004 ) . Meta Analysis of BACTEC MGIT 960 and BACTEC 460 T.B., with or without solid media, for detection of mycobacteria. J. Clin. Microbiol. 42, $2321-2325$.

Dye , C ., Scheele, S. Pathania, V., and Raviglione, M. (2003). Global burden of tuberculosis estimated incidence, prevalence and mortality by country. JAMA. $282,677-688$.
This increase in our study due to social state of human in this ages (Sharma and Mohan , 2004).

Ginsburg, A.S. Grosset, J.H. and Bishai , W.R. (2003). Fluoroquinolones, tuberculosis, and resistance. Lancet Infectious Diseases. 3, $432-442$.

Hanna , B., Ebrahimzadeh , A., Elliott , L., and Koyama, S. (2002) .Multicentral evaluation of the BACTEC MGIT 960 System for recovery of mycobacteria. J. Clin . Microbiol . 37, 748 - 752 .

Jayakumar, K . V., Forster , T., and Kyi , M. (2001) . Improved detection of Mycobacterium spp . using the BACTEC MGIT 960 System. Br. J . Biomed .Sci . 58, $154-158$.

Kent , P., and Kubica, G. (1985). Public health mycobacteriology : a guide for the level III laboratory. Department of Health and Human Services, Centers for Disease Control , Atlanta.

Kobayashi , I ., Toda, h., Koyama et al . (1999). Evaluation of Mycobacteria Growth Indicator Tube (MGIT), an automated culture system for detection of mycobacteria from clinical specimens.J.Clin. Microbiol.73, $172-178$.

Murugasu , B., and Dick, T. (2000). Bactericidal activity of nitrofurans against growing and dormant Mycobacterium bovis BCG. Journal of Antimicrobial Chemotherapy. 46, $917-919$.

Pereira , M., Tripathy, S. Inamdar , V., Ramesh, K., Bhavsar , M., and Risbud, A. (2005). Drug resistance pattern of Mycobacterium tuberculosis in seropositive and seronegative HIV - TB patients in Pune, India. Indian J. Med . Res . 121, 235239.

Rohner , P., Ninet, A., Benri , M., and Auckentaler, R. (2000). 
Evaluation of the BACTEC 960 automated nonradiometric system for isolation of mycobacteria from clinical specimens . Eur . J . Clin. Microbiol. Infect . Dis. 19, $715-717$.

Rooden, A.M. (2001). Isolation of Mycobacterium tuberculosis from patients with pulmonary tuberculosis and detection of primary and acquired resistance against anti - T.B drugs . Thesis. College of Science, University of Basrah , Iraq .
Sharma, S., and Mohan, A . (2004). Multidrug-resistance tuberculosis. Indian J . Med . Res . 120 , $354-376$.

Tazawa ,Y., Ishikawa, S., Furuhata, Y., and Okada, J. (1998) . Experience and clinical usefulness of BACTEC MGIT 960. J. Clin. Microbiol . 9, 59-65.

WHO (2006). Tuberculosis. Fast SHEET No. 104, Revised April 2006. GENEVA: World Health Organization .

\section{مقارنة اكتثاف جراثيمGycobacterium tuberculosis وباستخدام النظام الالي BACTEC MGIT 960 ووسط لوينشتاين جينشن}

$$
\text { محمد عبد الامام المازنية العلو / لباء بخعة البصرة الربيعي أياد عبد الكريم }
$$

اجريت الدراسة الحالية لاختبار فيما اذا كان نظام BACTEC MGIT 960 مككن استخدامه كبديل عن استخدام

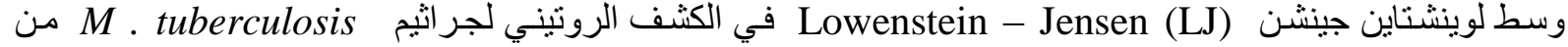

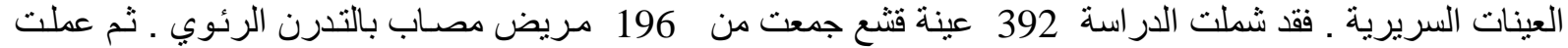
العينات بالطريقة القياسية المسمى بـ N - acetyl - L - Cysteine (NALC-NaOH) ولقحت بجهاز

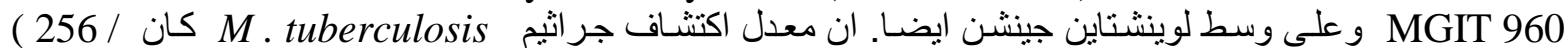

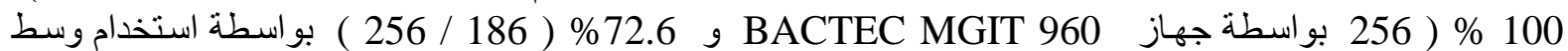

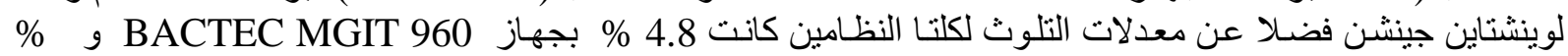

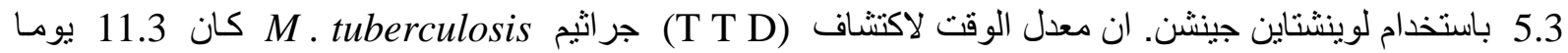

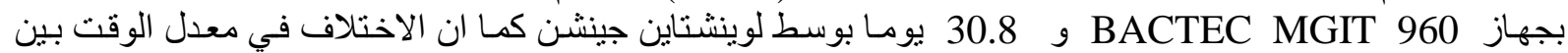

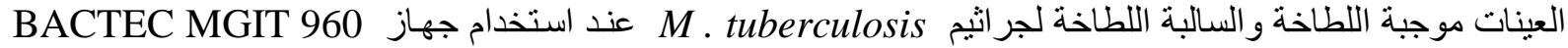

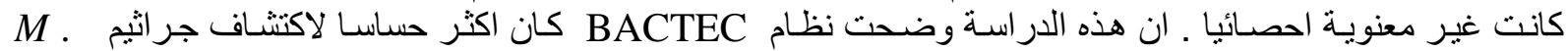
. tuberculosis 